\title{
A FLEXIBLE REGRESSION MODEL FOR COUNT DATA
}

\author{
By Kimberly F. Sellers And Galit Shmueli \\ Georgetown University and University of Maryland
}

\begin{abstract}
Poisson regression is a popular tool for modeling count data and is applied in a vast array of applications from the social to the physical sciences and beyond. Real data, however, are often over- or under-dispersed and, thus, not conducive to Poisson regression. We propose a regression model based on the Conway-Maxwell-Poisson (COM-Poisson) distribution to address this problem. The COM-Poisson regression generalizes the well-known Poisson and logistic regression models, and is suitable for fitting count data with a wide range of dispersion levels. With a GLM approach that takes advantage of exponential family properties, we discuss model estimation, inference, diagnostics, and interpretation, and present a test for determining the need for a COM-Poisson regression over a standard Poisson regression. We compare the COM-Poisson to several alternatives and illustrate its advantages and usefulness using three data sets with varying dispersion.
\end{abstract}

1. Introduction. Regression models are the most popular tool for modeling the relationship between a response variable and a set of predictors. In many applications, the response variable of interest is a count, that is, takes on nonnegative integer values. For count data, the most widely used regression model is Poisson regression, while, for binary data, the logistic (or probit) regression is most applied. Poisson regression is limiting in its variance assumption, namely, that for observation $i(i=1, \ldots, n), \operatorname{var}\left(Y_{i}\right)=E\left(Y_{i}\right)$. Even with the best of intent, however, count data often demonstrate over- or under-dispersion compared to the Poisson model.

One way to model over-dispersed count data is to use mixture models, for example, the gamma-Poisson mixture, where Poisson variables have means $\mu_{i}$ that follow a gamma distribution. This yields a negative binomial marginal distribution of the form

$$
P\left(Y_{i}=y_{i} \mid \mu_{i}, r\right)=\left(\frac{r}{r+\mu_{i}}\right)^{r} \frac{\Gamma\left(r+y_{i}\right)}{\Gamma\left(y_{i}+1\right) \Gamma(r)}\left(\frac{\mu_{i}}{r+\mu_{i}}\right)^{y_{i}}, \quad y_{i}=0,1,2, \ldots,
$$

where $r \geq 0$ and $\mu_{i} \geq 0$ for all $i(i=1, \ldots, n)$. The negative binomial likelihood can be expressed in the form of a generalized linear model for constant $r$, and a $\log$-link function $\left(\log \mu_{i}=\boldsymbol{\beta}^{\prime} \mathbf{X}_{i}\right)$ is typically used. Although negative binomial regression is available in many statistical software packages, it is limited to modeling only over-dispersed data. In addition to its inability to fit under-dispersed data,

Received December 2008; revised October 2009.

Key words and phrases. Conway-Maxwell-Poisson (COM-Poisson) distribution, dispersion, generalized linear models (GLM), generalized Poisson. 
McCullagh and Nelder (1997) note that this procedure is "an unpopular option with a problematic canonical link."

An alternative model which can capture both over- and under-dispersion is the restricted generalized Poisson regression (RGPR) model by Famoye (1993). The model is given by

$$
\begin{array}{r}
P\left(Y_{i}=y_{i} \mid \mu_{i}, \alpha\right)=\left(\frac{\mu_{i}}{1+\alpha \mu_{i}}\right)^{y_{i}} \frac{\left(1+\alpha y_{i}\right)^{y_{i}-1}}{y_{i} !} \exp \left(\frac{-\mu_{i}\left(1+\alpha y_{i}\right)}{1+\alpha \mu_{i}}\right), \\
y_{i}=0,1,2, \ldots,
\end{array}
$$

where $\log \mu_{i}=\boldsymbol{\beta}^{\prime} \mathbf{X}_{i}$. It is called a "restricted" model, because the dispersion parameter $\alpha$ is restricted to $1+\alpha \mu_{i}>0$ and $1+\alpha y_{i}>0$ [Cui, Kim and Zhu (2006)]. When $\alpha=0$, the model reduces to the Poisson case; $\alpha>0$ indicates over-dispersion; and $-2 / \mu_{i}<\alpha<0$ indicates under-dispersion. While this model allows for under- or over-dispersion in the data (albeit a limited degree of underdispersion), it belongs to an exponential family only for a constant dispersion parameter, $\alpha$. Thus, a more general model with observation-specific dispersion $\left(\alpha_{i}\right)$ will no longer belong to the exponential family. In short, for count data that are not binary nor follow a Poisson distribution, readily available, computationally efficient, flexible regression models are scarce. The need for such a model exists in many fields where count models are routinely fit to an array of data sets of varying dispersion.

In this paper we propose using a more general count distribution that captures a wide range of dispersion. A two-parameter generalized form of the Poisson distribution, called the Conway-Maxwell-Poisson (COM-Poisson) distribution [Shmueli et al. (2005)], is sufficiently flexible to describe a wide range of count data distributions. It includes as special cases the Poisson, Bernoulli, and geometric distributions, as well as distributions with dispersion levels between these three wellknown cases (governed by the dispersion parameter). The COM-Poisson distribution belongs to the exponential family and therefore possesses advantages in terms of estimation, conjugate priors, etc. These advantages have proven useful in several applications, such as using the COM-Poisson sufficient statistics for purposes of data disclosure [Kadane, Krishnan and Shmueli (2006)], in marketing applications [Boatwright, Borle and Kadane (2003), Borle et al. (2005)], and online auctions [Borle, Boatwright and Kadane (2006)]. We describe the COM-Poisson distribution and introduce a few additional COM-Poisson formulations in Section 2.

In Section 3 we use the COM-Poisson distribution to formulate a regression model. We discuss model estimation, inference, interpretation, and diagnostics; obtaining fitted values; and testing for dispersion. A Bayesian regression formulation using COM-Poisson has been used in marketing applications by Borle et al. (2005, 2007), Borle, Boatwright and Kadane (2006), Boatwright, Borle and Kadane (2003) and Kalyanam, Borle and Boatwright (2007). In each of these studies $\log (\lambda)$ was modeled as a linear function of predictors, and MCMC was used 
for estimation. Each of the data sets included a few thousand observations. For each model, estimation time was between 2-24 hours. Lord, Guikema and Geedipally (2008), motivated by traffic modeling, used a slightly different Bayesian formulation with $\log \left(\lambda^{1 / v}\right)$ as the link function. They use noninformative priors and their model yields good fit. The formulation used, however, does not take full advantage of the exponential family features of the COM-Poisson distribution and, in particular, requires computationally expensive MCMC for estimation. We, instead, approach the COM-Poisson distribution from a GLM perspective, carefully choosing a link function (namely, $\log \lambda$ ) that is advantageous in terms of estimation, inference, and diagnostics. Our formulation also creates a generalization of the ordinary Poisson regression as well as logistic regression, thereby including and bridging two very popular and well-understood models. Although the logistic regression is a limiting case $(v \rightarrow \infty)$, in practice, fitting a COM-Poisson regression to binary data yields estimates and predictions that are practically identical to those from a logistic regression.

To show the practical usefulness of the COM-Poisson regression, we compare its performance to a few alternative regression models: Poisson, negative binomial, logistic, and RGPR. Section 4 considers two data sets of different size and with different levels of dispersion. Using these data, we illustrate the advantages of the COM-Poisson model in terms of model fit, inference, and wide applicability. In Section 5 we consider the Lord, Guikema and Geedipally (2008) motor vehicle accidents example. We compare the models along with our COM-Poisson formulation to the Bayesian formulation. Section 6 concludes with discussion and future directions.

2. The COM-Poisson distribution. The COM-Poisson probability distribution function [Shmueli et al. (2005)] takes the form

$$
P\left(Y_{i}=y_{i}\right)=\frac{\lambda_{i}^{y_{i}}}{\left(y_{i} !\right)^{v} Z\left(\lambda_{i}, v\right)}, \quad y_{i}=0,1,2, \ldots, i=1, \ldots, n,
$$

for a random variable $Y_{i}$, where $Z\left(\lambda_{i}, v\right)=\sum_{s=0}^{\infty} \frac{\lambda_{i}^{s}}{(s !)^{v}}$ and $v \geq 0$. The ratio between the probabilities of two consecutive values is then $\frac{P\left(Y_{i}=y_{i}-1\right)}{P\left(Y_{i}=y_{i}\right)}=\frac{y_{i}^{\nu}}{\lambda_{i}}$. The COM-Poisson distribution generalizes the Poisson distribution in that the ratio is not necessarily linear in $y_{i}$, thereby leading to longer or shorter tails for the distribution. The COM-Poisson distribution includes three well-known distributions as special cases: Poisson $(v=1)$, geometric $\left(v=0, \lambda_{i}<1\right)$, and Bernoulli $\left(\nu \rightarrow \infty\right.$ with probability $\left.\frac{\lambda_{i}}{1+\lambda_{i}}\right)$.

In Shmueli et al. (2005) the moments are given in the form

$$
E\left(Y_{i}^{r+1}\right)= \begin{cases}\lambda_{i}\left[E\left(Y_{i}+1\right)\right]^{1-v}, & r=0, \\ \lambda_{i} \frac{\partial}{\partial \lambda_{i}} E\left(Y_{i}^{r}\right)+E\left(Y_{i}\right) E\left(Y_{i}^{r}\right), & r>0,\end{cases}
$$


and the expected value is approximated by

$$
E\left(Y_{i}\right)=\lambda_{i} \frac{\partial \log Z\left(\lambda_{i}, v\right)}{\partial \lambda_{i}} \approx \lambda_{i}^{1 / v}-\frac{v-1}{2 v} .
$$

In practice, the expected value can be evaluated by either (1) estimating the probability density function and truncating the infinite sum Minka et al. (2003), or (2) determining $\hat{\lambda}, \hat{v}$ and using these estimates to compute the approximation in Equation (2.2). Another useful result ${ }^{1}$ regarding this distribution is that $E\left(Y^{v}\right)=\lambda$. Note that the expected value and variance can also be written in the form

$$
\begin{aligned}
E\left(Y_{i}\right) & =\frac{\partial \log Z\left(\lambda_{i}, v\right)}{\partial \log \lambda_{i}}, \\
\operatorname{var}\left(Y_{i}\right) & =\frac{\partial E\left(Y_{i}\right)}{\partial \log \lambda_{i}} .
\end{aligned}
$$

We apply the results from equations (2.3) and (2.4) to formulate the estimating equations (available in the online supplemental materials) and the Fisher information matrix (Section 3).

3. Regression formulation. Our proposed COM-Poisson regression formulation begins as a generalization of an ordinary Poisson regression. McCullagh and Nelder (1997) view Poisson regression as a special case of loglinear models taking the form

$\log E\left(Y_{i}\right)=\log \mu_{i}=\eta_{i}=\boldsymbol{\beta}^{\prime} \mathbf{X}_{i}=\beta_{0}+\beta_{1} X_{i 1}+\cdots+\beta_{p} X_{i p}, \quad i=1, \ldots, n$,

where $\operatorname{var}\left(Y_{i}\right)=\sigma^{2} E\left(Y_{i}\right)$, and where $\sigma^{2}$ denotes the dispersion parameter $\left[\sigma^{2}>1\right.$ $(<1)$ for over- (under) dispersion]. Further, they argue that the link function is more important than the variance assumption. We will show that, while in some cases dispersion might not significantly affect mean predictions, it does affect the conditional distributions and can affect inference.

We can write a similar approximate type of relationship between the mean and variance via the COM-Poisson distribution. Using equations (2.1)-(2.2), we can write (suppressing subscript $i$ )

$$
\operatorname{var}(Y)=\lambda \frac{\partial}{\partial \lambda} E(Y) \approx \lambda \frac{\partial}{\partial \lambda}\left(\lambda^{1 / v}-\frac{v-1}{2 v}\right)=\frac{1}{v} \lambda^{1 / v} \approx \frac{1}{v} E(Y),
$$

in accordance with McCullagh and Nelder (1997). Thus, we can see the relationship between $v$ (or $\frac{1}{v}$ ) and the direction of data dispersion.

In the following we take a more direct approach to modeling the dispersion by extending the GLM formulation to the COM-Poisson case and modeling the relationship between $Y$ and the predictors $\mathbf{X}$ via a function of $E(Y)$. Although typical

\footnotetext{
${ }^{1}$ We thank Ralph Snyder for providing this result.
} 
link functions are direct functions of $E(Y)$ [e.g., $E(Y), \log E(Y), \operatorname{logit}(E(Y))]$, the most natural link function for a COM-Poisson regression is $\eta(E(\mathbf{Y}))=\log \lambda$, modeling the relationship between $E(\mathbf{Y})$ and $\mathbf{X}$ indirectly. This choice of function is useful for two reasons. First, it coincides with the link function in two wellknown cases: in Poisson regression, it reduces to $E(\mathbf{Y})=\lambda$; in logistic regression, where $\mathbf{p}=\frac{\lambda}{\mathbf{1}+\lambda}$, it reduces to $\operatorname{logit}(\mathbf{p})=\log \lambda$. The second advantage of using $\log \lambda$ as the link function is that it leads to elegant estimation, inference, and diagnostics. This result highlights the lesser role that the conditional mean plays when considering count distributions of a wide variety of dispersion levels. Unlike Poisson or linear regression, where the conditional mean is central to estimation and interpretation, in the COM-Poisson regression model, we must take into account the entire conditional distribution.

\subsection{Model estimation. We write the log-likelihood for observation $i$ as}

$$
\log L_{i}\left(\lambda_{i}, v \mid y_{i}\right)=y_{i} \log \lambda_{i}-v \log y_{i} !-\log Z\left(\lambda_{i}, v\right) .
$$

Summing over $n$ observations, the log-likelihood is given by

$$
\log L=\sum_{i=1}^{n} y_{i} \log \lambda_{i}-v \sum_{i=1}^{n} \log y_{i} !-\sum_{i=1}^{n} \log Z\left(\lambda_{i}, v\right) .
$$

Maximum likelihood coefficient estimates can be obtained by directly maximizing equation (3.2) under the constraint $v \geq 0$, using a constrained nonlinear optimization tool (e.g., nlminb in R). An alternative is to write the log-likelihood as a function of $\log v$, and then maximize it using an ordinary nonlinear optimization tool (e.g., nlm in R). A third option for obtaining the maximum likelihood estimates is to use the GLM framework to formulate the likelihood maximization as a weighted least squares procedure (see online supplemental material) and to solve it iteratively.

The GLM formulation is also used for deriving standard errors associated with the estimated coefficients. The latter are derived using the Fisher information matrix. For estimating $\boldsymbol{\beta}$ and $\nu$, we have a block Information matrix of the form

$$
\mathbf{I}=\left(\begin{array}{cc}
\mathbf{I}^{\boldsymbol{\beta}} & \mathbf{I}^{\boldsymbol{\beta}, v} \\
\mathbf{I}^{\boldsymbol{\beta}, v} & I^{v}
\end{array}\right)
$$

where $\mathbf{I}^{\boldsymbol{\beta}}$ pertains to the estimated variances and covariances of $\hat{\boldsymbol{\beta}}, I^{\nu}$ contains the estimated variance for $\hat{v}$, and $\mathbf{I}^{\boldsymbol{\beta}, v}$ contains the componentwise estimates of the covariance between $\hat{\boldsymbol{\beta}}$ and $\hat{v}$. Details regarding the information matrix components are available in the online supplementary material. $\mathrm{R}$ code for estimating COM-Poisson regression coefficients and standard errors is available at www9.georgetown.edu/faculty/kfs7/research. 
3.2. Testing for dispersion. How much data dispersion should exist to warrant deviation from Poisson regression? The set of hypotheses, $H_{0}: v=1$ vs. $H_{1}$ : $v \neq 1$, ask whether the use of Poisson regression is reasonable versus the alternative of fitting COM-Poisson regression. Note that $H_{1}$ does not specify the direction (over vs. under) of data dispersion. This can be assessed, however, via exploratory data analysis and the dispersion estimate, $\hat{v}$, from the fitted COM-Poisson regression.

We derive the test statistic,

$$
C=-2 \log \Lambda=-2\left[\log L\left(\hat{\boldsymbol{\beta}}^{(0)}, \hat{v}=1\right)-\log L(\hat{\boldsymbol{\beta}}, \hat{v})\right],
$$

where $\Lambda$ is the likelihood ratio test statistic, $\hat{\boldsymbol{\beta}}^{(0)}$ are the maximum likelihood estimates obtained under $H_{0}: v=1$ (i.e., the Poisson estimates), and $(\hat{\boldsymbol{\beta}}, \hat{v})$ are the maximum likelihood estimates under the general state space for the COM-Poisson distribution. Under the null hypothesis, $C$ has an approximate $\chi^{2}$ distribution with 1 degree of freedom. For small samples, the test statistic distribution can be estimated via bootstrap.

3.3. Computing fitted values. Once a COM-Poisson regression model has been estimated, we can obtain fitted values $\left(\hat{y}_{i}\right)$ in one of two ways:

1. Estimated means: We can use the approximation in equation (2.2) and obtain fitted values by $\hat{y}_{i} \mid \mathbf{x}_{i}=\hat{\lambda}_{i}^{1 / \hat{v}}-\frac{\hat{v}-1}{2 \hat{v}}$, where $\hat{\lambda}_{i}=\exp \left(\mathbf{x}_{i}^{\prime} \hat{\boldsymbol{\beta}}\right)$. Note that this approximation is accurate for $v \leq 1$ or $\lambda_{i}>10^{v}$ [Minka et al. (2003)].

2. Estimated medians: When the mean approximation is inadequate (or in general), we can obtain percentiles of the fitted distribution by using the inverseCDF for $\hat{y}_{i} \mid \mathbf{x}_{i}$ and $\hat{v}$. In particular, we use the estimated median to obtain fitted values.

3.4. Model inference. Due to the GLM formulation, the statistical significance of individual predictors can be obtained by using the asymptotic standard normal distribution of $\hat{\beta}_{j} / \hat{\sigma}_{\hat{\beta}_{j}}$. In the case of small samples, however, where the asymptotic normality might not hold (as in other count data regression models), bootstrapping can be used to estimate the distributions of the coefficients of interest. With small samples, COM-Poisson model estimation is very fast, thereby being practically useful for bootstrap.

A parametric COM-Poisson bootstrap can be implemented by resampling from a COM-Poisson distribution with parameters $\hat{\boldsymbol{\lambda}}=\exp \left(\mathbf{X}^{\prime} \hat{\boldsymbol{\beta}}\right)$ and $\hat{v}$, where $\hat{\boldsymbol{\beta}}, \hat{v}$ are estimated from a COM-Poisson regression on the full data set. The resampled data sets include new $Y$ values accordingly. Then, for each resampled data set, a COMPoisson regression is fit, thus producing new associated estimates, which can then be used for inference. 
3.5. Coefficient interpretation. There are two main approaches for interpreting coefficients in regression models [Long (1997)]. One examines changes in the conditional mean for a unit increase in a single predictor, for example, $E\left(Y \mid X_{j}=\right.$ $\left.x_{j}, \mathbf{X}_{i \neq j}=\mathbf{x}\right)$ and $E\left(Y \mid X_{j}=x_{j}+1, \mathbf{X}_{i \neq j}=\mathbf{x}\right)$. In additive models, such as a linear regression, the difference between the two conditional means [or the derivative of $E(Y \mid X)$ with respect to $X_{j}$ ] is used for interpretation ["a unit increase in $X_{j}$ is associated with a $\beta_{j}$ increase in $E(Y)$ "']; in multiplicative models, such as the Poisson or logistic regressions, the ratio of the two conditional means is used for interpretation ["a unit increase in $X_{j}$ is associated with a factor of $e^{\beta_{j}}$ increase in $E(Y)$ or the odds"]. The second approach, which is used for coefficient interpretation in other types of nonlinear regression models (e.g., probit regression), is to directly examine the relationship between fitted values and changes in a predictor. This can be done via graphical plots for less than two predictors, while, for more than two predictors, there are various solutions such as fitted value consideration at selected values of the predictors.

In the COM-Poisson regression case, we cannot use the first approach that compares conditional means directly, because the relationship between the conditional mean and the predictors is neither additive nor multiplicative (except for the special cases of Poisson and logistic regressions). For example (considering a single predictor model), the ratio of conditional means leads to a complicated nonlinear relationship between a unit increase in $X$ and the effect on $E(Y \mid X)$. However, the result $E\left(Y^{v}\right)=\lambda$ in Section 2 indicates a multiplicative relationship between the predictors and $E\left(Y^{v}\right)$. It appears, however, that interpreting the effect of individual predictors on the conditional mean (or median) directly is most straightforward via the second approach.

Because coefficients from a COM-Poisson regression model are on a different scale than those from an ordinary Poisson model, for purposes of crude comparison, one can simply divide the COM-Poisson coefficients by $v$. This approach is reasonable because $E\left(\mathbf{Y}^{v}\right)=\lambda$.

3.6. Model diagnostics. Due to the GLM formulation and, in particular, the IWLS framing (see online supplemental material), standard GLM diagnostics can be used for residual analysis of a fitted COM-Poisson regression model. We use the matrices $\mathcal{W}$ and $\mathcal{X}$ as defined there for computing leverage, and the popular Pearson and Deviance residuals. Leverage can be computed from the hat matrix, $H=\mathcal{W}^{1 / 2} \mathcal{X}\left(\mathcal{X}^{\prime} \mathcal{W} \mathcal{X}\right)^{-1} \mathcal{X}^{\prime} \mathcal{W}^{1 / 2}$. An observation with an unusually high value of $h_{i}$ is suspect of having influence (although $H$, like other nonlinear models, depends on the estimated parameters). Meanwhile, using ordinary GLM formulations, we can write the Pearson residual for observation $i$ [Davison and Tsai (1992)] as $r_{P, i}=\frac{Y_{i}-\hat{\mu}_{i}}{\sqrt{w_{i}\left(1-h_{i}\right)}}$, where $\hat{\mu}_{i}=\widehat{E\left(Y_{i}\right)}$, and the standardized deviance residual for observation $i$ can be written as $r_{D, i}=\operatorname{sgn}\left(Y_{i}-\hat{\mu}_{i}\right) \frac{d_{i}}{\sqrt{1-h_{i}}}$, where $d_{i}=-2\left[\log L\left(\hat{\mu}_{i}, y_{i} ; \hat{v}\right)-\log L\left(y_{i}, y_{i} ; \hat{v}\right)\right]$. These two types of residuals can 
be computed directly or approximated using the mean approximation in Equation (2.2). In particular, for deviance residuals, the approximation leads to

$$
\begin{aligned}
d_{i}=2\left[y_{i} \hat{v} \log \left(\left(y_{i}+\frac{\hat{v}-1}{2 \hat{v}}\right) /\left(\hat{\mu}_{i}+\frac{\hat{v}-1}{2 \hat{v}}\right)\right)\right. \\
\left.\quad+\log \left(Z\left(\left(\hat{\mu}_{i}+\frac{\hat{v}-1}{2 \hat{v}}\right)^{\hat{v}}, \hat{v}\right) / Z\left(\left(y_{i}+\frac{\hat{v}-1}{2 \hat{v}}\right)^{\hat{v}}, \hat{v}\right)\right)\right] .
\end{aligned}
$$

The existence of equation (3.4) is constrained in that $Y>k$ for $\hat{v}<\frac{1}{2 k+1} ; k \in \mathbf{N}^{+}$. We can, however, modify equation (3.4) in order to obtain valid results for $d_{i}$. For example, when $v<1$ and $Y=0$, we set $Z\left(\left(y_{i}+\frac{\hat{v}-1}{2 \hat{v}}\right)^{\hat{v}}, \hat{v}\right)=1$. Another option is to use the exact deviance equations supplied above, though this is computationally more expensive. Finally, while the approximation is accurate for $\lambda>10^{v}$ or $v<1$, we have found that deviance residuals computed using equation (3.4) are quite accurate even outside that range (e.g., for under-dispersed data with low counts).

A probability plot of the deviance residuals, as well as a scatter plot of $\log (\hat{\lambda})$ versus deviance residuals, can help assess model adequacy and detect outliers. Although normal probability plots are common, deviance residuals for nonlinear models can be far from normally distributed [Ben and Yohai (2004)]. One alternative is to ignore the fit to normality on the normal probability plot, and use it just to detect outliers. Another option is to use bootstrap to estimate the distribution of deviance residuals, and then to create a QQ plot of the deviance residuals against their estimated distribution.

4. Examples. In this section we fit regression models to data sets characterized by under-dispersion, and with binary outcomes (i.e., extreme underdispersion); Section 5 discusses the over-dispersion example considered by Lord, Guikema and Geedipally (2008). We fit various popular regression model choices for count data: Poisson, negative binomial (NB), restricted generalized Poisson (RGPR), and COM-Poisson. For the binary data set, we also fit a logistic regression. The goal of this section is to compare the COM-Poisson to the other models in terms of fit, inference, and flexibility. The small sample size and dimension of the first data set is useful for directly observing the effect of dispersion. In particular, we show the effect of dispersion on the conditional distribution of fit. We evaluate goodness-of-fit and predictive power by examining the fitted values and comparing values of MSE and $\mathrm{AIC}_{C}$ (the Akaike Information Criterion ${ }^{2}$ corrected for small sample size) across models.

Note that, except for the Poisson and logistic regressions, the other models considered have an extra dispersion parameter that is assumed fixed across observations, but unknown. Each of the models is estimated by maximum likelihood. The

\footnotetext{
${ }^{2}$ All models aside from Poisson have a penalty term in the $\mathrm{AIC}_{C}$ that takes into account the extra dispersion parameter.
} 
Poisson, NB, and logistic regressions are estimated using ordinary GLM functions in R. COM-Poisson is estimated using nonlinear optimization in R, and standard errors are estimated as described in Section 3.1. RGPR is estimated using constrained nonlinear optimization in $\mathrm{R}$ and standard errors are estimated as described in Famoye (1993).

4.1. Regression with under-dispersed data: Airfreight breakage. We first consider the airfreight breakage example from [Kutner, Nachtsheim and Neter (2003), page 35, Exercise 1.21] where data are given on 10 air shipments, each carrying 1000 ampules on the flight. For each shipment $i$, we have the number of times the carton was transferred from one aircraft to another $\left(X_{i}\right)$ and the number of ampules found broken upon arrival $\left(Y_{i}\right)$. The data are provided online among the supplementary material.

We first estimated the COM-Poisson regression coefficients and tested for dispersion. The estimated dispersion parameter is $\hat{v}=5.78$, indicating underdispersion. To test for dispersion, we use parametric bootstrap (see Section 3.4) rather than the dispersion test, due to the small sample size. The $90 \%$ bootstrap confidence interval for $v$ is $(4.00,21.85)$, indicating dispersion that requires a COM-Poisson regression instead of ordinary Poisson regression. We proceed by attempting to fit the four regression models. The estimated coefficients and standard errors for three of these models (Poisson, NB, and COM-Poisson) are given in Table 1; NB regression produces identical estimates to that from Poisson regression. RGPR did not converge and, therefore, no estimated model is produced. This highlights the limited ability of RGPR to fit under-dispersed data. In general, for under-dispersed data, the RGPR probability function "gets truncated and does not necessarily sum to one" [Famoye, Wulu and Singh (2004)]. This example appears to fall exactly under this limitation.

Fitted values from the models are provided online in the supplementary material where, for the COM-Poisson, we use the estimated conditional median for fitted values because the approximation (2.2) is likely to be inaccurate (here, $v>1$ and $\lambda \ngtr 10^{v}$ ). We find that the models are similar in terms of the fitted values that they generate (see also Figure 1). In terms of MSE and $\mathrm{AIC}_{C}$, the COM-Poisson

TABLE 1

Estimated coefficients and standard errors (in parentheses) for the airfreight example, for various regression models. NB and Poisson regression produce the same estimates. The RGPR did not converge

\begin{tabular}{lrr}
\hline Model & \multicolumn{1}{c}{$\hat{\boldsymbol{\beta}}_{\mathbf{0}}\left(\hat{\boldsymbol{\sigma}}_{\hat{\boldsymbol{\beta}}_{\mathbf{0}}}\right)$} & $\hat{\boldsymbol{\beta}}_{\mathbf{1}}\left(\hat{\boldsymbol{\sigma}}_{\hat{\boldsymbol{\beta}}_{\mathbf{1}}}\right)$ \\
\hline Poisson/NB & $2.3529(0.1317)$ & $0.2638(0.0792)$ \\
COM-Poisson $\left(\hat{v}=5.7818, \hat{\sigma}_{\hat{v}}=2.597\right)$ & $13.8247(6.2369)$ & $1.4838(0.6888)$ \\
\hline
\end{tabular}




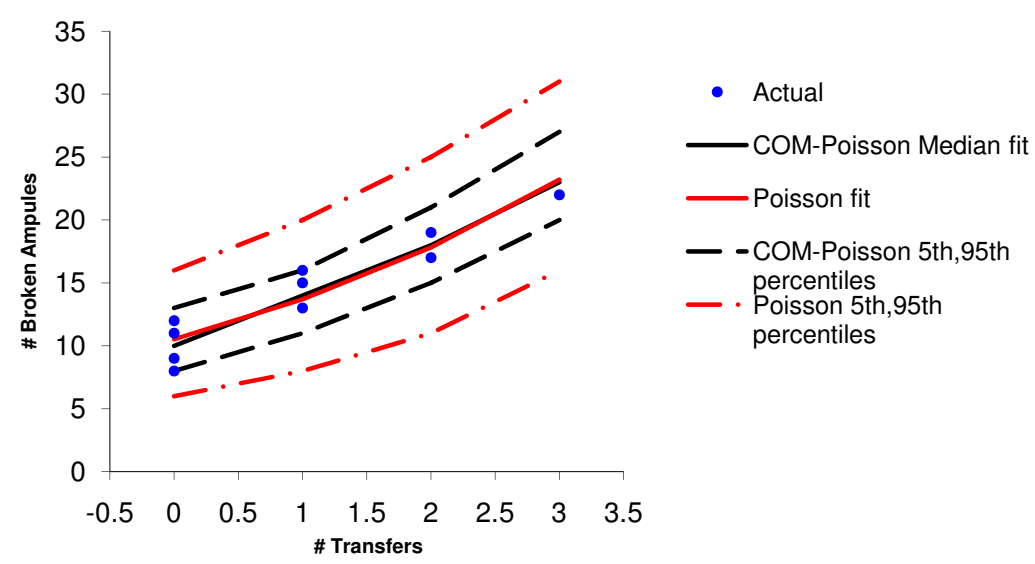

FIG. 1. Fitted mean curves (solid lines), 5th and 95th percentile curves (broken lines) for Poisson and COM-Poisson regression models for the airfreight breakage data (dots).

shows best fit, although the differences between models for these values are not large (see Table 2). The similarity of the regression models is also in terms of the coefficient magnitudes (after dividing the COM-Poisson coefficients by $\hat{v}$ ). The models differ, however, in two important ways. First, although the fitted values are similar, the conditional distribution differs markedly across the models, as can be seen by comparing the 5th and 95th percentile curves in Figure 1. Second, the models initially appear to differ in terms of inference. Comparing the Poisson, and COM-Poisson estimated models, we find that the ratio $\hat{\beta}_{1} / \hat{\sigma}_{\hat{\beta}_{1}}$ is 3.33 and 2.15 , respectively. Due to the small sample size, however, the normal approximation might not be adequate. We therefore examined the distributions of $\hat{\beta}_{0}$ and $\hat{\beta}_{1}$ for each of the models, based on 1000 parametric bootstrapped samples (see Section 3.4). Figure 2 displays normal probability plots for the estimated coefficients. We see that the distributions for the COM-Poisson model are skewed. To evaluate statistical significance of the predictor (number of transfers), we examine the percent of the distribution of $\hat{\beta}_{1}$ to the left of the value $\beta_{1}=0$. In both models, this percent is zero, indicating high statistical significance.

TABLE 2

Airfreight breakage example: goodness-of-fit and predictive power statistics

\begin{tabular}{lcc}
\hline & $\begin{array}{c}\text { COM-Poisson } \\
\text { median fit }\end{array}$ & $\begin{array}{c}\text { Poisson } \\
\text { fit }\end{array}$ \\
\hline $\mathrm{AIC}_{C}$ & $\mathbf{4 7 . 2 9}$ & 52.11 \\
$\mathrm{MSE}$ & $\mathbf{1 . 9 0}$ & 2.21 \\
\hline
\end{tabular}


Beta0, Poisson

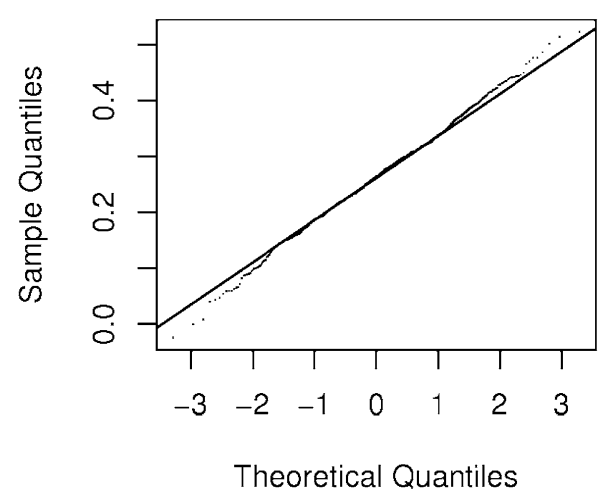

Beta1, Poisson

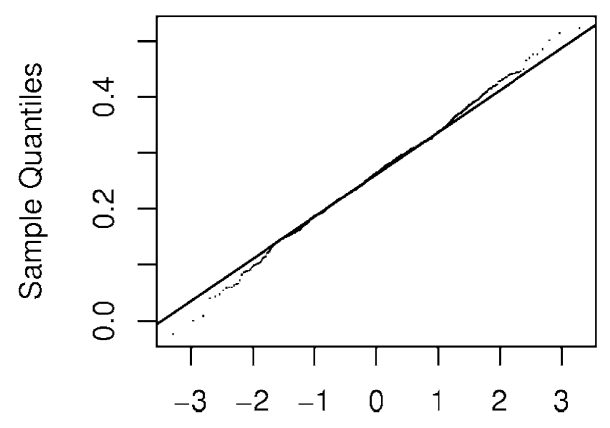

Theoretical Quantiles
Beta0, COM-Poisson

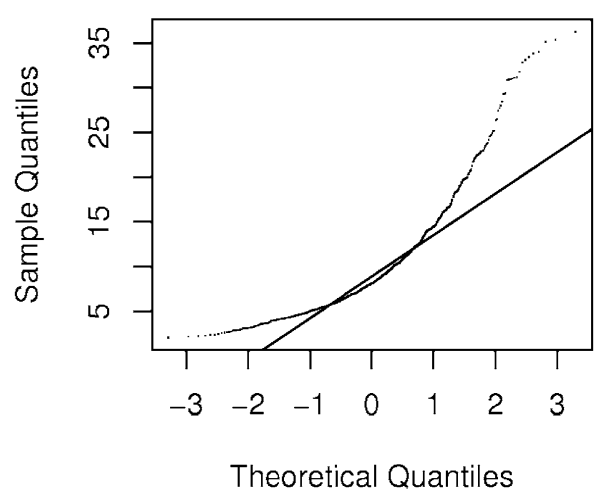

Beta1, COM-Poisson

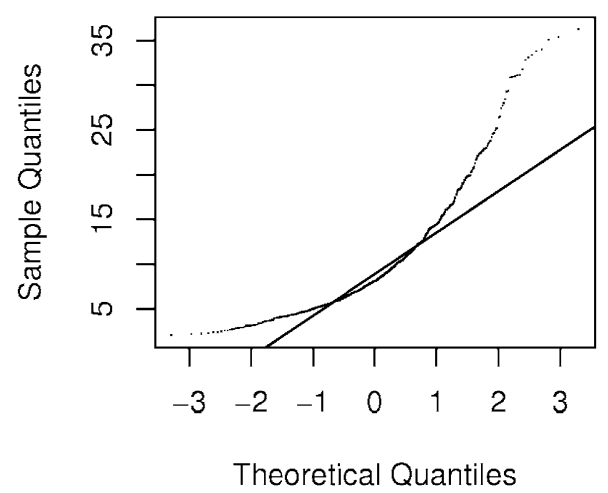

FIG. 2. Normal probability plots of $\hat{\beta}_{0}$ (top) and $\hat{\beta}_{1}$ (bottom) based on 1000 bootstrap samples of the airfreight breakage data. Negative binomial estimation produces identical results to those from Poisson regression. RGPR estimation procedure does not converge.

In terms of model interpretation, the Poisson regression indicates that a unit increase in the number of transfers is associated with a factor increase of 1.3 in the average number of broken ampules. Looking at Figure 1, however, shows that interpretations in terms of the average number of broken ampules is insufficient. In particular, the number of transfers seems to affect the entire distribution of the number of broken ampules, as indicated by the fitted COM-Poisson model. Indeed, the COM-Poisson curves in Figure 1 can be used directly for interpreting the relationship between number of transfers and number of broken ampules.

Finally, we examine leverage and scaled deviance residuals from each of the models. Figure 3 displays scatterplots of the deviance residuals versus the single predictor (which is equivalent to plotting versus $\log \hat{\lambda}$ for the Poisson and COM- 

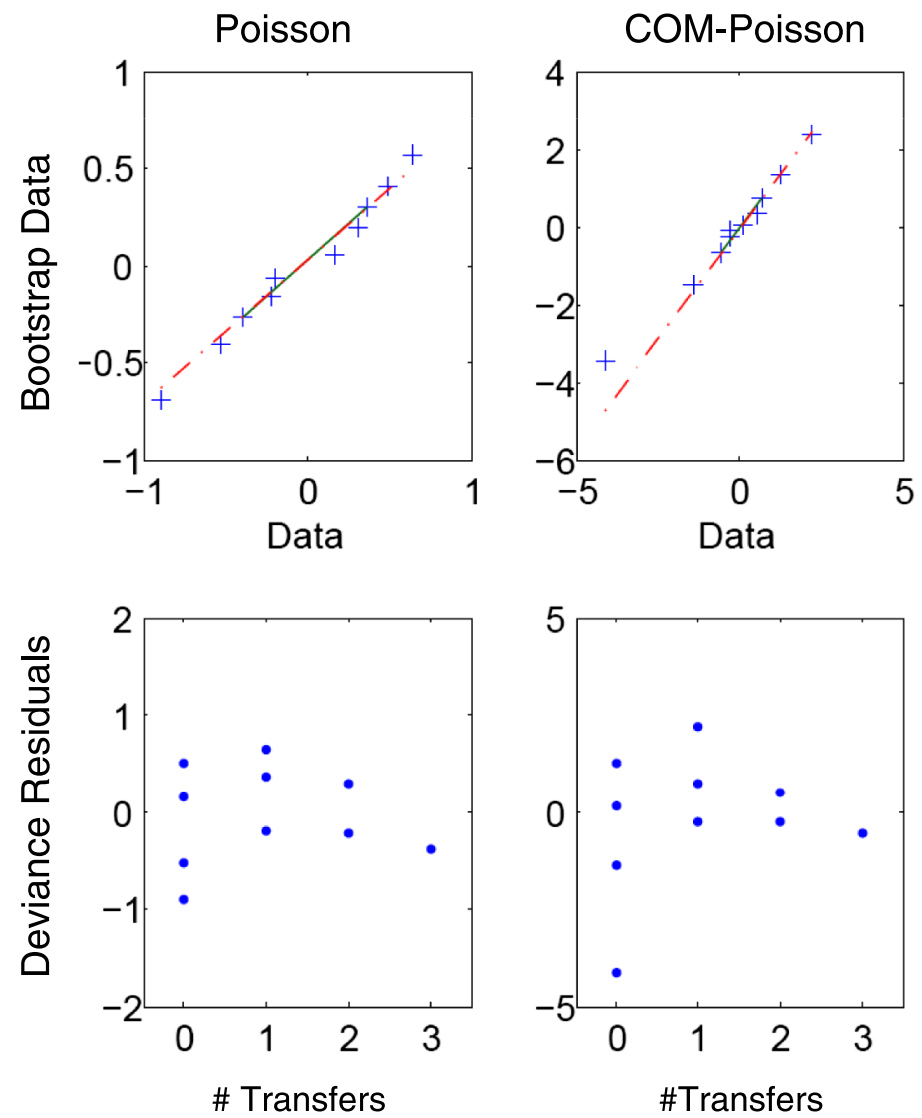

FIG. 3. QQ plots of the scaled deviance residuals (top) and scatterplots of the scaled deviance residuals vs. the predictor (bottom) for the airfreight breakage data. Each column corresponds to a different regression model.

Poisson models), and QQ plots. Leverage values are available in the online supplementary materials. Overall, there is no noticeable pattern in any of the scatterplots. Both models indicate observation \#5 (with $X=3$ ) as suspect of being influential, and observation $\# 7$ as an outlier (having a large negative deviance residual), particularly for the COM-Poisson model.

4.2. Regression with extreme under-dispersion: Book purchases. We now consider the case where the outcome variable is binary, and where typically a logistic regression would have been considered. Although the logistic regression is theoretically only a limiting case of the COM-Poisson regression, we show that (in practice) a fitted COM-Poisson to binary outcome data produces practically identical results to a logistic regression. We use a data set from Lattin, Green and Caroll (2003) that describes the results of a direct-marketing campaign by a book club, 
TABLE 3

Estimated coefficients and standard errors (in parentheses) for Book Club example, for four regression models (NB estimates are identical to Poisson; RGPR did not converge). The estimates for the logistic and COM-Poisson models are identical, even to eight decimal places

\begin{tabular}{lccc}
\hline Model & $\hat{\boldsymbol{\beta}}_{\mathbf{0}}\left(\hat{\boldsymbol{\sigma}}_{\hat{\boldsymbol{\beta}}_{\mathbf{0}}}\right)$ & $\hat{\boldsymbol{\beta}}_{\text {Months }}\left(\hat{\boldsymbol{\sigma}}_{\hat{\boldsymbol{\beta}}_{\text {Months }}}\right)$ & $\hat{\boldsymbol{\beta}}_{\text {ArtBooks }}\left(\hat{\boldsymbol{\sigma}}_{\hat{\boldsymbol{\beta}}_{\text {ArtBooks }}}\right)$ \\
\hline Poisson/NB & $-2.29(0.18)$ & $-0.06(0.02)$ & $0.73(0.05)$ \\
Logistic & $-2.23(0.24)$ & $-0.07(0.02)$ & $0.99(0.14)$ \\
COM-Poisson $\quad\left(\hat{v}=30.4, \hat{\sigma}_{\hat{v}}=10,123\right)$ & $-2.23(0.24)$ & $-0.07(0.02)$ & $0.99(0.14)$ \\
\hline
\end{tabular}

for a certain art book. ${ }^{3}$ The data set contains the results for 1000 customers. The outcome is whether the customer purchased the art book or not. The two predictor variables are the number of months since the customer's last purchase (Months), and the number of art books that the customer has purchased in the past (ArtBooks). We use this data set to show the flexibility of the COM-Poisson regression over the alternatives discussed above. In particular, we show that the COM-Poisson regression produces estimates and predictions that are identical (to multiple decimals) to those from a logistic regression, and that RGPR and NB fail to converge altogether.

Table 3 provides the parameter estimates from the Poisson, logistic, and COMPoisson regression models, respectively. The NB regression estimates are identical to the Poisson estimates. RGPR is absent from Table 3 because it has limited ability to capture under-dispersion, thus, it fails to converge.

With respect to comparing COM-Poisson with logistic regression, it is clear that the two models produce identical results in terms of coefficients and standard errors (even to eight decimals). Meanwhile, we note the large estimated value for $v$, along with its broad standard error. This is in congruence with the terms of the COM-Poisson distribution for the special case of a Bernoulli random variable (namely, $v \rightarrow \infty$ ). Furthermore, comparing fitted values (or predictions), using the estimated COM-Poisson median as the fitted value (in accordance with Section 3.3) yields values that are identical to those from a logistic regression with cutoff value 0.5 . To obtain fits for other cutoff values, the corresponding percentile should be used. Finally, although the Poisson model does converge, it is clearly inappropriate in terms of inference, and produces fitted values that are not binary.

5. Regression with over-dispersed data: Modeling motor vehicle crashes. The previous section shows the flexibility of the COM-Poisson regression to capture under-dispersion, which exceeds the ability of models such as the negative binomial and RGPR. We now examine an over-dispersed data set used by Lord,

\footnotetext{
${ }^{3}$ Two additional examples where COM-Poisson regression is applied to binary data (showing similar results) are given in the online supplemental materials.
} 
Guikema and Geedipally (2008) which contains motor vehicle crash data in 1995 , at 868 signalized intersections located in Toronto, Ontario. For each intersection, measurements included the annual number of crashes at the intersection $(Y)$ and two traffic flow variables. See Lord, Guikema and Geedipally (2008) for further details on the data.

Because motor vehicle crash data contain counts, Poisson and negative binomial regressions are common models in the field of transportation safety. For the Toronto data set, Lord, Guikema and Geedipally (2008) proposed using a Bayesian COM-Poisson regression formulation to model the over-dispersion. In particular, they used noninformative priors and modeled the effect of the two traffic variables on the number of crashes via the link function $\log \left(\lambda^{1 / \nu}\right)=\mathbf{X} \boldsymbol{\beta}$. Parameter estimation was then performed via MCMC. The authors note that estimation for this data set used 35,000 replications, requiring nearly five hours of computation. Comparing goodness-of-fit and out-of-sample prediction measures, Lord, Guikema and Geedipally (2008) showed the similarity in performance of the COM-Poisson and negative binomial regression. They then motivate the advantage of the COMPoisson over the negative binomial regression in the ability to fit under-dispersion and low counts.

The goal of this section is two-fold: (1) to extend the model comparison in Lord, Guikema and Geedipally (2008) beyond the negative binomial model to additional models, as well as to examine a wider range of model comparison aspects, and (2) to compare the Bayesian COM-Poisson formulation to our formulation and show the advantages gained by using our formulation. Although goodness-offit measures might indicate similarity of the COM-Poisson performance to other models, model diagnostics provide additional information.

5.1. Model estimation. Various regression models were fit to the Toronto intersection crash data. Following Lord, Guikema and Geedipally (2008), the response was the number of crashes at the intersection, and the two covariates were the two log-transformed traffic flow variables.

Table 4 displays the estimated models: two COM-Poisson formulations [our model and the Bayesian model of Lord, Guikema and Geedipally (2008)], and three alternative regression models (Poisson, NB, and RGPR). From $\hat{v}<1$ and $\hat{\alpha}>0$, over-dispersion is indicated. All $\hat{\beta}$ coefficients appear similar across the models. For standard errors, the Poisson estimates are much smaller than in other models (as expected in over-dispersion).

Comparing the two COM-Poisson formulations, the two are nearly identical in terms of $\hat{v}$ and its standard error [or the equivalent posterior credible standard error for Lord, Guikema and Geedipally (2008)], and in terms of the $\hat{\beta}$ coefficients (after scaling by a factor of $\hat{v}$, due the different formulation of the relationship between the covariates and the response). These similarities between the Bayesian and classic formulations indicate that the prior information does not affect the model, here most likely due to the large size of the data set. The most dramatic 
TABLE 4

Estimated models: comparing two COM-Poisson formulations [ours and Lord, Guikema and Geedipally (2008)], and alternative models for the Toronto crash data. For ease of comparison, we report the COM-Poisson estimates and standard errors from our formulation in terms of $\hat{v}$ multipliers, to reflect the comparable scale with estimates from the other models

\begin{tabular}{|c|c|c|c|c|}
\hline Model & Extra parameter & $\hat{\beta}_{0}\left(\hat{\sigma}_{\hat{\beta}_{0}}\right)$ & $\hat{\beta}_{1}\left(\hat{\sigma}_{\hat{\beta}_{1}}\right)$ & $\hat{\beta}_{2}\left(\hat{\sigma}_{\hat{\beta}_{2}}\right)$ \\
\hline Our formulation & $\hat{v}=0.3492(0.0208)$ & $-11.7027 \hat{v}(0.7501 \hat{v})$ & $0.6559 \hat{v}(0.0619 \hat{v})$ & $0.7911 \hat{v}(0.0461 \hat{v})$ \\
\hline $\begin{array}{l}\text { Lord, Guikema } \\
\text { and Geedipally } \\
\text { (2008) }\end{array}$ & $\hat{v}=0.3408(0.0208)$ & $-11.53(0.4159)$ & $0.6350(0.0474)$ & $0.7950(0.0310)$ \\
\hline Poisson & & $-10.2342(0.2838)$ & $0.6029(0.0288)$ & $0.7038(0.0140)$ \\
\hline Neg-Bin & $\hat{r}=7.154(0.625)$ & $-10.2458(0.4626)$ & $0.6207(0.0456)$ & $0.6853(0.0215)$ \\
\hline RGPR & $\hat{\alpha}=0.050(0.004)$ & $-10.2357(0.4640)$ & $0.6205(0.0451)$ & $0.6843(0.0215)$ \\
\hline
\end{tabular}

difference between the two implementations is in run time: our estimation took less than three minutes, compared to five hours required by the Bayesian MCMC. This difference has significance especially since Lord, Guikema and Geedipally (2008) used noninformative priors to obtain their estimates. Thus, in the absence of strong prior information or in the presence of a large data set, our formulation provides more efficient estimation. Even in the presence of prior information, our method is still useful for obtaining initial estimates to speed up the MCMC process.

5.2. Model performance. Comparing goodness-of-fit measures, the two COM-Poisson formulations are practically identical in terms of $\hat{\beta}$ and thus produce nearly identical fitted values. Compared to the other regression models, the COM-Poisson model has lower MSE and AIC values, indicating better fit and predictive power (see Table 5). The COM-Poisson dispersion test (with $C=518$, and associated $p$-value $=0$ ) indicates that the COM-Poisson model is more adequate than Poisson regression.

We now examine model diagnostics to better understand model fit. Figure 4 displays scatterplots of the scaled deviance residuals vs. $\log \hat{\lambda}$. For RGPR, we use unscaled deviance residuals (as $H$ is unavailable). From the residual plots and the leverage measures (available in the online supplementary materials), we find that the NB model marks nearly half of the $Y=0$ observations as influential, and

TABLE 5

Goodness-of-fit comparison of COM-Poisson with alternative fitted models

\begin{tabular}{lcccc}
\hline & COM-Poisson & Poisson & Neg-Bin & RGPR \\
\hline AIC & $\mathbf{5 0 7 3}$ & 5589 & 5077 & 5092 \\
MSE & $\mathbf{3 2 . 5 7}$ & 32.60 & 32.70 & 32.71 \\
\hline
\end{tabular}




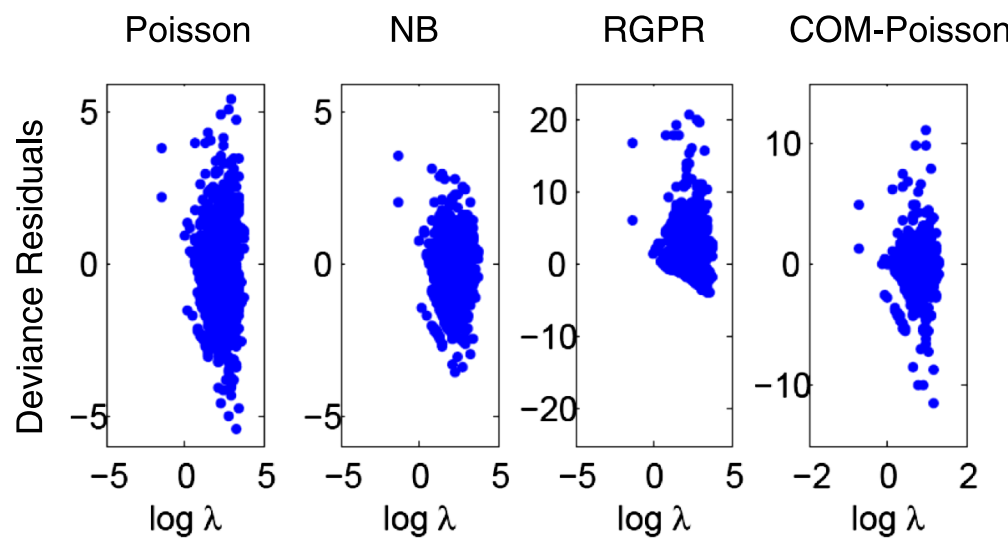

FIG. 4. Scatterplots of the scaled deviance residuals vs. $\log \hat{\lambda}$. Each column corresponds to a different regression model. For RGPR the deviance residuals are unscaled.

flags mostly high-count observations. The Poisson and NB models mark the observations with largest $X$ values as influential. In contrast, COM-Poisson diagnostics point out eight observations with large residuals (\#15, \#42, \#247, \#424, \#494, \#618, \#619, \#757) and three with high leverage (\#133, \#801, \#835). Three of the large-residual intersections have a large number of crashes with relatively little traffic (small values of the covariates). The remaining large-residual intersections have a small to medium number of crashes, but less substantial traffic on one of the traffic flow covariates. All of these observations are also flagged by at least one other regression method, with observations \#15 and \#618 being flagged by all methods.

5.3. Inference. In terms of drawing inference about the effect of the traffic flow covariates on the number of crashes, we examine the coefficients and standard errors and assume a normal approximation. In this case, the effects are very strong across all models, resulting in $p$-values of zero for each of the two covariate coefficients.

6. Discussion. The COM-Poisson regression model provides a practical tool for modeling count data that have various levels of dispersion. It generalizes the widely-used Poisson regression, as well as allows for other levels of dispersion. Using a GLM approach and taking advantage of the exponential family properties of the COM-Poisson distribution, we provide a straightforward, elegant, computationally efficient framework for model estimation, dispersion testing, inference, and diagnostics. The data examples illustrate the differences and similarities that arise in practice when using a COM-Poisson regression versus more traditional regression models. For moderate to high counts, fitted values can be similar across models but the conditional fitted distribution can differ markedly. Models also tend 
to diverge in terms of inference for single predictors, implying that inappropriate use of a Poisson model (instead of a COM-Poisson model) can lead to erroneous conclusions.

One important insight from the COM-Poisson regression model is that, in a model that allows for different levels of dispersion, the role of the conditional mean is no longer central. Unlike linear regression or Poisson regression where the conditional mean is central to interpretation, the COM-Poisson regression uses a more general function of the response distribution. The resulting model means that, when examining goodness-of-fit or when predicting new observations, the complete conditional fitted distribution must be taken into account rather than just the conditional mean.

The elegance of the COM-Poisson regression model lies in its ability to address applications containing a wide range of dispersion in a parsimonious way. While the negative binomial model is a popular resource for count data applications where over-dispersion exists, it cannot address problems where data are under-dispersed. The RGPR formulation offers more flexibility in its ability to handle data dispersion, yet it is limited in the level of under-dispersion that it can capture. We have shown that, in such cases, the COM-Poisson regression does not encounter such difficulties and produces reasonable fitted models. The COMPoisson regression has the flexibility even in the extreme case of a binary response, where it reduces to a logistic regression in theory, and produces identical estimates and predictors in practice.

Our regression model is similar to the Bayesian formulation used by Borle et al. (2005, 2007), Borle, Boatwright and Kadane (2006), Boatwright, Borle and Kadane (2003), Kalyanam, Borle and Boatwright (2007) and that by Lord, Guikema and Geedipally (2008) in terms of the generated estimated parameters. It differs from the Bayesian formulation, however, both conceptually [in terms of the link function of Lord, Guikema and Geedipally (2008) and the estimation method] and practically (with regard to run time). Although the Bayesian implementation allows for the incorporation of prior information in the form of prior parameter distributions [e.g., see Kadane et al. (2005)], the benefit of such information is useful only when informative priors are used and when the sample size is small. Second, specifying meaningful priors on the $\beta$ coefficients is not straightforward, as it requires an understanding of the function $\lambda^{1 / v}$, which is not equal to the mean. Software implementation also differentiates these models because our formulation relies on traditional estimation methods for exponential family distributions: estimation, inference, and diagnostics can be programmed in most statistical software packages in a straightforward manner. From a computational point of view, although the $Z$ function requires approximation (because it is an infinite sum), in practice, a simple truncation of the sum performs well.

A potential restricting factor in our current COM-Poisson regression formulation is that it assumes a constant dispersion level across all observations. This is similar to the classic homoscedasticity assumption in linear regression. A possible 
enhancement is to allow $v$ to be observation-dependent (and to model it as a function of covariates as well). In our COM-Poisson regression formulation such an extension still maintains the structure of an exponential family, unlike that of the generalized Poisson regression of Famoye (1993), for example.

The relationship between the associated fitted mean bands and the estimated data dispersion is nicely illustrated in accordance with McCullagh and Nelder (1997). Further work is needed to investigate their impact on Type I errors associated with hypothesis testing about the slope, or slope coverage. In addition, this work introduces several questions regarding sample size, which, although easily overcome by using bootstrap, present interesting research questions.

Finally, while not presented in this work, simulations were performed to demonstrate the accuracy of the estimation process, as well as that of the hypothesis testing procedure. $\mathrm{R}$ code for simulating COM-Poisson data is also available at www9.georgetown.edu/faculty/kfs $7 /$ research.

Acknowledgments. The authors thank Seth Guikema and Dominique Lord for supplying the Toronto crash data to allow for method comparison. The authors also thank Jay Kadane and the reviewers for their helpful and insightful comments.

\section{SUPPLEMENTARY MATERIAL}

Supplementary Materials: (DOI: 10.1214/09-AOAS306SUPP). Materials include details of the iterative reweighted least squares estimation, the Fisher information matrix components associated with the COM-Poisson coefficients, the full airfreight data set and diagnostics under various regression models for the airfreight and crash data, and additional logistic regression examples.

\section{REFERENCES}

Ben, M. G. and YoHAI, V. J. (2004). Quantile quantile plot for deviance residuals in the generalized linear model. J. Comput. Graph. Statist. 13 36-47. MR2044869

Boftwright, P., Borle, S. and Kadane, J. B. (2003). A model of the joint distribution of purchase quantity and timing. J. Amer. Statist. Assoc. 98 564-572. MR2011672

Borle, S., Boftwright, P. and Kadane, J. B. (2006). The timing of bid placement and extent of multiple bidding: An empirical investigation using ebay online auctions. Statist. Sci. 21 194-205. MR2324078

Borle, S., Boatwright, P., Kadane, J. B., Nunes, J. C. and Shmueli, G. (2005). The effect of product assortment changes on customer retention. Marketing Science 24 616-622.

Borle, S., Dholakia, U., Singh, S. and Westbrook, R. (2007). The impact of survey participation on subsequent behavior: An empirical investigation. Marketing Science 26 711-726.

CUI, Y., KIM, D.-Y. and ZHU, J. (2006). On the generalized Poisson regression mixture model for mapping quantitative trait loci with count data. Genetics 174 2159-2172.

DAVISON, A. and TSAI, C.-L. (1992). Regression model diagnostics. International Statistical Review $60337-353$.

FAmoYe, F. (1993). Restricted generalized Poisson regression model. Comm. Statist. Theory Methods 22 1335-1354. MR1225247 
Famoye, F., Wulu, J. J. and K. P. Singh (2004). On the generalized Poisson regression model with an application to accident data. Journal of Data Science 2 287-295.

KADANe, J. B., Krishnan, R. and Shmueli, G. (2006). A data disclosure policy for count data based on the COM-Poisson distribution. Management Science 52 1610-1617.

Kadane, J. B., Shmueli, G., Minka, T. P., Borle, S. and Boatwright, P. (2005). Conjugate analysis of the Conway-Maxwell-Poisson distribution. Bayesian Anal. 1363-374. MR2221269

Kalyanam, K., Borle, S. and Boatwright, P. (2007). Deconstructing each item's category contribution. Marketing Science 26 327-341.

Kutner, M. H., Nachtsheim, C. J. and Neter, J. (2003). Applied Linear Regression Models, 4th ed. McGraw-Hill, New York.

Lattin, J. M., Green, P. E. J. and Caroll, D. (2003). Analyzing Mulivariate Data. Duxbury, Pacific Grove, CA.

Long, J. S. (1997). Regression Models for Categorical and Limited Dependent Variables. Sage, London.

Lord, D., Guikema, S. D. and GeedipalLy, S. R. (2008). Application of the Conway-MaxwellPoisson generalized linear model for analyzing motor vehicle crashes. Accident Analysis \& Prevention 40 1123-1134.

McCullagh, P. and Nelder, J. A. (1997). Generalized Linear Models, 2nd ed. Chapman \& Hall/CRC, London. MR0727836

Minka, T. P., Shmueli, G., Kadane, J. B., Borle, S. and Boatwright, P. (2003). Computing with the COM-Poisson distribution. Technical Report 776, Dept. Statistics, Carnegie Mellon Univ., Pittsburgh, PA.

Shmueli, G., Minka, T. P., Kadane, J. B., Borle, S. and Boatwright, P. (2005). A useful distribution for fitting discrete data: Revival of the Conway-Maxwell-Poisson distribution. Appl. Statist. 54 127-142. MR2134602

DEPARTMENT OF MATHEMATICS

GEORGETOWN UNIVERSITY

WASHINGTON, DC 20057

USA

E-MAIL: kfs7@georgetown.edu
Department of Decision, Operations \& INFORMATION TECHNOLOGIES

SMITH SCHOOL OF BUSINESS

UNIVERSITY OF MARYLAND

College Park, MARYLAND 20742

USA

E-MAIL: gshmueli@rhsmith.umd.edu 